\title{
1 Global diversity and geography of soil fungi
}

3 Leho Tedersoo $^{1 * \dagger}+$, Mohammad Bahram $^{2} \dagger$, Sergei Põlme ${ }^{1}$, Urmas Kõljalg $^{2}$, Nourou S. Yorou ${ }^{3}$,

4 Ravi Wijesundera ${ }^{4}$, Luis Villarreal Ruiz ${ }^{5}$, Aída M. Vasco-Palacios ${ }^{6}$, Pham Quang Thu ${ }^{7}$, Ave

5 Suija $^{2}$, Matthew E. Smith ${ }^{8}$, Cathy Sharp $^{9}$, Erki Saluveer $^{2}$, Alessandro Saitta $^{10}$, Miguel Rosas ${ }^{11}$,

6 Taavi Riit ${ }^{2}$, David Ratkowsky ${ }^{12},{\text { Karin } \text { Pritsch }^{13}, \text { Kadri Põldmaa }^{2}, \text { Meike Piepenbring }}^{11}$,

7 Cherdchai Phosri ${ }^{14}$, Marko Peterson ${ }^{2}$, Kaarin Parts $^{2}$, Kadri Pärtel $^{2}$, Eveli Otsing ${ }^{2}$, Eduardo

8 Nouhra $^{15}$, André L. Njouonkou ${ }^{16}$, R. Henrik Nilsson ${ }^{17}$, Luis N. Morgado ${ }^{18}$, Jordan Mayor ${ }^{19}$,

9 Tom W. May ${ }^{20}$, Luiza Majuakim ${ }^{21}$, D. Jean Lodge ${ }^{22}$, Su See Lee ${ }^{23}$, Karl-Henrik Larsson ${ }^{24}$, Petr

10 Kohout $^{2}$, Kentaro Hosaka ${ }^{25}$, Indrek Hiiesalu ${ }^{2}$, Terry W. Henkel ${ }^{26}$, Helery Harend $^{2}$, Liang-dong

$11 \mathrm{Guo}^{27}$, Alina Greslebin ${ }^{28}$, Gwen Grelet ${ }^{29}$, Jozsef Geml ${ }^{18}$, Genevieve Gates ${ }^{12}$, William

12 Dunstan $^{30}$, Chris Dunk $^{19}$, Rein Drenkhan ${ }^{31}$, John Dearnaley $^{32}$, André De Kesel $^{33}$, Tan Dang ${ }^{7}$,

13 Xin Chen $^{34}$, Franz Buegger ${ }^{13}$, Francis Q. Brearley ${ }^{35}$, Gregory Bonito ${ }^{20}$, Sten Anslan ${ }^{2}$, Sandra

14 Abell $^{36}$, Kessy Abarenkov ${ }^{2}$

$16{ }^{1}$ Natural History Museum, University of Tartu, Tartu, Estonia.

$17 \quad{ }^{2}$ Institute of Ecology and Earth Sciences, University of Tartu, Tartu, Estonia.

$18{ }^{3}$ Faculté d'Agronomie, Université de Parakou, Parakou, Benin.

$19{ }^{4}$ Department of Plant Sciences, University of Colombo, Colombo 3, Sri Lanka.

$20{ }^{5}$ Postgrado en Recursos Genéticos y Productividad-Genética, LARGEMBIO, Colegio de

21 Postgraduados-LPI 6, México City, Mexico.

$22{ }^{6}$ The Fungal Biodiversity Centre, CBS-KNAW, Utrecht, The Netherlands.

$23{ }^{7}$ Vietnamese Academy of Forest Sciences, Hanoi, Vietnam. 
$24{ }^{8}$ Department of Plant Pathology, University of Florida, Gainesville, Florida, USA.

$25{ }^{9}$ Natural History Museum, Bulawayo, Zimbabwe.

$26{ }^{10}$ Department of Agricultural and Forest Sciences, Università di Palermo, Palermo, Italy.

$27{ }^{11}$ Department of Mycology, Goethe University Frankfurt, Frankfurt am Main, Germany.

$28 \quad{ }^{12}$ Tasmanian Institute of Agriculture, Hobart, Tasmania, Australia.

$29{ }^{13}$ Institute of Soil Ecology, Helmholtz Zentrum München, Neuherberg, Germany.

$30{ }^{14}$ Department of Biology, Nakhon Phanom University, Nakhon Phanom, Thailand.

$31{ }^{15}$ Instituto Multidisciplinario de Biología Vegetal, Córdoba, Argentina.

$32{ }^{16}$ Department of Biological Sciences, University of Bamenda, Bambili, Cameroon.

$33{ }^{17}$ Department of Biological and Environmental Sciences, University of Gothenburg, Göteborg,

34 Sweden.

$35{ }^{18}$ Naturalis Biodiversity Center, Leiden, The Netherlands.

$36{ }^{19}$ Department of Forest Ecology and Management, Swedish University of Agricultural

37 Sciences, Umeå, Sweden.

$38{ }^{20}$ Royal Botanic Gardens Melbourne, Melbourne, Victoria, Australia.

$39{ }^{21}$ Institute for Tropical Biology and Conservation, University Malaysia Sabah, Sabah,

40 Malaysia.

$41 \quad{ }^{22}$ Center for Forest Mycology Research, USDA-Forest Service, Luquillo, Puerto Rico.

$42 \quad{ }^{23}$ Forest Research Institute Malaysia, Kepong, Selangor, Malaysia.

$43 \quad{ }^{24}$ Natural History Museum, University of Oslo, Oslo, Norway.

$44 \quad{ }^{25}$ Department of Botany, National Museum of Nature and Science, Tsukuba, Japan.

$45{ }^{26}$ Department of Biological Sciences, Humboldt State University, Arcata, California, USA. 
${ }^{27}$ State Key Laboratory of Mycology, Institute of Microbiology, Chinese Academy of Sciences,

47 Beijing, China.

$48{ }^{28}$ CONICET - Facultad de Cs. Naturales, Universidad Nacional de la Patagonia SJB, Esquel,

49 Chubut, Argentina.

$50 \quad{ }^{29}$ Ecosystems and Global Change team, Landcare Research, Lincoln, New Zealand.

$51{ }^{30}$ School of Veterinary \& Life Sciences, Murdoch University, Western Australia, Australia.

$52{ }^{31}$ Institute of Forestry and Rural Engineering, Estonian University of Life Sciences, Tartu,

53 Estonia.

$54{ }^{32}$ Faculty of Health, Engineering and Sciences, University of Southern Queensland,

55 Toowoomba, Queensland, Australia.

$56 \quad{ }^{33}$ Botanic Garden Meise, Meise, Belgium.

$57 \quad{ }^{34}$ College of Life Sciences, Zhejiag University, Hangzhou 310058, China.

$58{ }^{35}$ School of Science and the Environment, Manchester Metropolitan University, Manchester,

59 United Kingdom.

$60{ }^{36}$ School of Marine and Tropical Biology, James Cook University, Cairns, Queensland,

61 Australia.

62

$63 \uparrow$ Equal contribution

$64 *$ Corresponding author. E-mail: leho.tedersoo@ut.ee

65 
68 Fungi play major roles in ecosystem processes, but the determinants of fungal diversity and

69 biogeographic patterns remain poorly understood. By using DNA metabarcoding data from

70 hundreds of globally distributed soil samples, we demonstrate that fungal richness is decoupled

71 from plant diversity. The plant-to-fungus richness ratio declines exponentially towards the

72 poles, indicating strong biases in previous fungal diversity estimates. Climatic factors,

73 followed by edaphic and spatial variables, constitute the best predictors of fungal richness and

74 community composition at the global scale. Fungi follow general biogeographic patterns

75 related to latitudinal diversity gradients but with several notable exceptions. These findings

76 significantly advance our understanding of fungal diversity patterns at the global scale and

77 permit integration of fungi into a general macro-ecological framework.

80 One-sentence summary

82 A massive, global-scale metagenomic study detects hotspots of fungal diversity and

83 macroecological patterns, and indicates that plant and fungal diversity are uncoupled. 
86 INTRODUCTION: The kingdom Fungi is one of the most diverse groups of organisms on

87 Earth and they are integral ecosystem agents that govern soil carbon cycling, plant nutrition,

88 and pathology. Fungi are widely distributed in all terrestrial ecosystems, but the distribution of

89 species, phyla, and functional groups has been poorly documented. Based on 365 global soil

90 samples from natural ecosystems, we determined the main drivers and biogeographic patterns

91 of fungal diversity and community composition.

92 RATIONALE: We identified soil-inhabiting fungi using 454 pyrosequencing and comparison

93 against taxonomically and functionally annotated sequence databases. Multiple regression

94 models were used to disentangle the roles of climatic, spatial, edaphic, and floristic parameters

95 on fungal diversity and community composition. Structural equation models were used to

96 determine the direct and indirect effects of climate on fungal diversity, soil chemistry and

97 vegetation. We also examined if fungal biogeographic patterns matched paradigms derived

98 from plants and animals - namely, that species' latitudinal ranges increase towards the poles

99 (Rapoport's rule) and diversity increases towards the equator. Finally, we sought group-

100 specific global biogeographic links among major biogeographic regions and biomes using a

101 network approach and area-based clustering.

102 RESULTS: Metabarcoding analysis of global soils revealed fungal richness estimates

103 approaching the number of species recorded to date. Distance from equator and mean annual

104 precipitation had the strongest effects on richness of fungi including most fungal taxonomic

105 and functional groups. Diversity of most fungal groups peaked in tropical ecosystems, but

106 ectomycorrhizal fungi and several fungal classes were most diverse in temperate or boreal

107 ecosystems and many fungal groups exhibited distinct preferences for specific edaphic

108 conditions (e.g. pH, calcium, phosphorus). Consistent with Rapoport's rule, the geographic 
range of fungal taxa increased toward the poles. Fungal endemicity was particularly strong in

110 tropical regions, but multiple fungal taxa had cosmopolitan distribution.

111 CONCLUSIONS: Climatic factors, followed by edaphic and spatial patterning, are the best

112 predictors of soil fungal richness and community composition at the global scale. Richness of

113 all fungi and functional groups is causally unrelated to plant diversity with the exception of

114 ectomycorrhizal root symbionts, suggesting that plant-soil feedbacks do not influence the

115 diversity of soil fungi at the global scale. The plant-to-fungi richness ratio declined

116 exponentially towards the poles, indicating that current predictions assuming globally constant

117 ratios overestimate fungal richness by 1.5-2.5-fold. Fungi follow similar biogeographic

118 patterns as plants and animals with the exception of several major taxonomic and functional

119 groups that run counter to overall patterns. Strong biogeographic links among distant

120 continents reflect relatively efficient long-distance dispersal compared with macro-organisms.

122 Figure caption

123 Direct and indirect effects of climatic and edaphic variables on plant and fungal richness.

124 Line thickness corresponds to relative path coefficients. Dashed lines indicate negative 125 relationships. Abbreviations: MAP, mean annual precipitation; Fire, time since last fire.

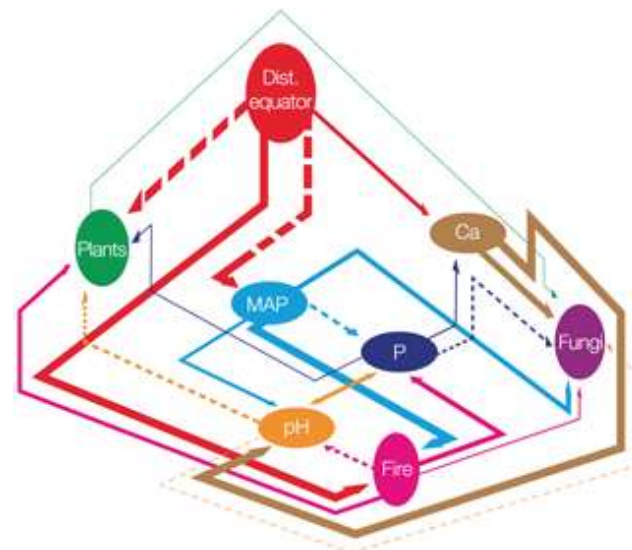


129 Fungi are eukaryotic microorganisms that play fundamental ecological roles as decomposers, 130 mutualists, or pathogens of plants and animals; they drive carbon cycling in forest soils, 131 mediate mineral nutrition of plants, and alleviate carbon limitations of other soil organisms.

132 Fungi comprise some 100,000 described species (accounting for synonyms), but the actual 133 extent of global fungal diversity is estimated at 0.8 to 5.1 million species (1).

Globally, the biomass and relative proportions of microbial groups, including fungi, co-

135 vary with the concentration of growth-limiting nutrients in soils and plant tissues. Such

136 patterns suggest that the distribution of microbes reflects latitudinal variation in ecosystem

137 nutrient dynamics (2-4). Richness of nearly all terrestrial and marine macro-organisms is 138 negatively related to increasing latitude (5) - a pattern attributed to the combined effects of 139 climate, niche conservatism, and rates of evolutionary radiation and extinction (6). Although 140 morphological species of unicellular microbes are usually cosmopolitan (7), there is growing 141 evidence that the distribution of micro-organisms is shaped by macro-ecological and

142 community assembly processes (8). Only a few of these biogeographic processes have been 143 demonstrated for fungi at the local scale (9). Despite their enormous diversity and importance 144 in ecosystem function, little is known about general patterns of fungal diversity or functional 145 roles over large geographic scales. Here we use a global dataset to disentangle the roles of 146 climatic, edaphic, floristic, and spatial variables governing global-scale patterns of soil fungal 147 diversity. We also address macro-ecological phenomena and show that fungi largely exhibit 148 strong biogeographic patterns that appear to be driven by dispersal limitation and climate. 
150 Materials and Methods

152 Sample preparation

153 We collected 40 soil cores from natural communities in each of 365 sites across the world

154 using a uniform sampling protocol (Fig. 1A; Data S1). Most plots (2500 $\left.\mathrm{m}^{2}\right)$ were circular, but

155 in steep mountain regions and densely forested areas, some plots were oblong. We randomly

156 selected twenty trees located at least $8 \mathrm{~m}$ apart. In two opposite directions, 1-1.5 $\mathrm{m}$ from each

157 tree trunk, loose debris was removed from the forest floor. PVC tubes $(5 \mathrm{~cm}$ diam. $)$ were

158 hammered into the soil down to $5 \mathrm{~cm}$ depth. These soil cores almost always included fine roots

159 and comprised both the organic layer and top mineral soil. Although deep soil may contain

160 some unique organisms adapted to anoxic conditions or low nutrient levels, our sampling was

161 limited to topsoil for the following reasons. First, in the vast majority of soil types, $>50 \%$ of

162 microbial biomass and biological activity occur in the topmost organic soil layer. Second,

163 deeper sampling was impossible in shallow, rocky soils or those with high clay concentrations

164 and hardpans. Third, differences among soil horizons may be masked by other variables across

165 large geographic scales (10). The 40 soil cores taken in each site were pooled, coarse roots and

166 stones removed, and a subset of the soil was air-dried at $<35^{\circ} \mathrm{C}$. Dried soil was stored in zip-

167 lock plastic bags with silica gel to minimize humidity during transit. In the laboratory, dried

168 soil was ground into fine powder using bead beating.

169 DNA was extracted from $2.0 \mathrm{~g}$ of soil using the PowerMax Soil DNA Isolation kit

170 (MoBio, Carlsbad, CA USA) following manufacturer's instructions. PCR was performed using

171 a mixture of six forward primers (in equimolar concentration) analogous to ITS3 and a 
172 degenerate reverse primer analogous to ITS4 (hereafter referred to as ITS4ngs). Forward and

173 reverse primers were shortened and modified to completely match $>99.5 \%$ of all fungi (except

174 ca. $60 \%$ of Tulasnellaceae that exhibit highly divergent 5.8S rDNA and Microsporidia that

175 exhibit re-arrangements in ribosomal DNA; Table S1). The ITS4ngs primer was tagged with

176 one of 110 identifiers (MIDs, 10-12 bases) that were modified from those recommended by

177 Roche to differ by $>3$ bases, start only with adenosine, and consist of between $30-70 \%$

178 adenosine and thymidine in order to optimize the adapter ligation step. The PCR cocktail

179 consisted of $0.6 \mu \mathrm{l}$ DNA extract, $0.5 \mu \mathrm{l}$ each of the primers (20 pmol), $5 \mu \mathrm{l} 5 \mathrm{xHOT}$ FIREPol

180 Blend Master Mix (Solis Biodyne, Tartu, Estonia), and $13.4 \mu$ l double-distilled water. PCR

181 was carried out in four replicates using the following thermocycling conditions: an initial 15

$182 \mathrm{~min}$ at $95^{\circ} \mathrm{C}$, followed by 30 cycles at $95{ }^{\circ} \mathrm{C}$ for $30 \mathrm{~s}, 55^{\circ} \mathrm{C}$ for $30 \mathrm{~s}, 72{ }^{\circ} \mathrm{C}$ for $1 \mathrm{~min}$, and a

183 final cycle of $10 \mathrm{~min}$ at $72{ }^{\circ} \mathrm{C}$. PCR products were pooled and their relative quantity was

184 estimated by running $5 \mu \mathrm{l}$ amplicon DNA on $1 \%$ agarose gel for $15 \mathrm{~min}$. DNA samples

185 yielding no visible band were re-amplified using 35 cycles in an effort to obtain sufficient PCR

186 product, whereas samples with a very strong band were re-amplified with only 25 cycles. It is

187 important to use as few cycles as possible to minimize chimera formation and to be able to 188 interpret sequence abundance in a semiquantitative manner (11). We used negative (for DNA

189 extraction and PCR) and positive controls throughout the experiment. Amplicons were purified 190 with Exonuclease I and FastAP thermosensitive alkaline phosphatase enzymes (Thermo

191 Scientific, Pittsburgh, PA USA). Purified amplicons were subjected to quantity normalization

192 with a SequalPrep Normalization Plate Kit (Invitrogen, Carlsbad, CA, USA) following

193 manufacturer's instructions. Normalized amplicons were divided into five pools that were

194 subjected to 454 adaptor ligation, emulsion PCR, and 454 pyrosequencing using the GS-FLX+ 
technology and Titanium chemistry as implemented by Beckman Coulter Genomics (Danvers,

196 MA, USA).

198 Bioinformatics

199 Pyrosequencing on five half-plates resulted in 2,512,068 reads with a median length of 409

200 bases. The sequences were re-assigned to samples in mothur 1.32.2 (www.mothur.org) based

201 on the barcodes and then trimmed (parameters: minlength $=300$; maxambigs $=1$;

202 maxhomop=12; qwindowaverage=35; qwindowsize $=50$; bdiffs $=1$ ) to exclude short and low-

203 quality sequences, resulting in 2,231,188 high quality sequences. We used ITSx 1.0.7

204 (http://microbiology.se/software/itsx) to remove the flanking 5.8S and 28S rRNA genes for 205 optimal resolution of ITS2 clustering and removal of compromised and non-target sequences.

206 As a filter to remove most of the partial sequences we retained only sequences $>99$ bp in 207 length. Chimera control was exercised through UCHIME 4.2 (www.drive5.com/uchime/).

208 After these filtering steps, 1,397,679 sequences were retained and further clustered at 90.0\% 209 and 95.0-99.0\% sequence similarity thresholds (12) as implemented in CD-Hit 4.6.1 (www.cd210 hit.org). Clustering revealed 37,387, 59,556, 66,785, 77,448, 94,255, and 157,956 taxa based 211 on $90.0 \%, 95.0 \%, 96.0 \%, 97.0 \%, 98.0 \%$, and $99.0 \%$ sequence similarity thresholds,

212 respectively. The longest sequence of each Operational Taxonomic Unit (OTU), based on 213 clustering at $98.0 \%$ sequence similarity, was selected as the representative for BLASTn 214 searches (word size=7; penalties: gap=-1; gap extension=-2; match=1) against the International 215 Nucleotide Sequence Databases Collaboration (INSDC: www.insdc.org) and UNITE 216 (unite.ut.ee) databases. In addition, we ran BLASTn searches against established reference 217 sequences of all fungi in $99.0 \%$ similarity clusters that include third-party taxonomic and 
218 metadata updates (12) as implemented in the PlutoF workbench (13). For each query, we

219 considered the 10 best-matching references to annotate our global sequences as accurately as

220 possible. If no reliable taxon name was available, we ran manual BLASTn searches against

221 INSDC with 500 best matching sequences as output. We typically relied on $90 \%, 85 \%, 80 \%$,

222 and $75 \%$ sequence identity as a criterion for assigning OTUs with names of a genus, family,

223 order, or class, respectively. Sequence identity levels were raised in subsets of

224 Sordariomycetes, Leotiomycetes, and Eurotiomycetes, because these taxa contain multiple

225 genera and families that have unusually conserved ITS sequences. As a rule, we considered e-

226 values of BLASTn search results $<\mathrm{e}^{-50}$ reliable to assign sequences to the fungal kingdom,

227 whereas those $>\mathrm{e}^{-20}$ were considered 'unknown'. E-values between $\mathrm{e}^{-20}$ and $\mathrm{e}^{-50}$ were manually

228 checked against the 10 best matches for accurate assignment. We followed INSDC for higher-

229 level taxonomy of eukaryotes (14) and the Index Fungorum (www.indexfungorum.org) for

230 species through class-level taxonomy of fungi. Our group of taxonomic experts assigned each

231 fungal genus, family, or order to functional categories (Data S2). If different functional

232 categories were present within a specific genus, we chose the dominant group ( $>75 \%$ of

233 species assigned to a specific category) or considered its ecology unknown ( $<75 \%$ of species

234 assignable to a single category). All Glomeromycota were considered to be arbuscular

235 mycorrhizal (AM). Taxa were considered to be ectomycorrhizal (EcM) if they best matched

236 any sequences of known EcM lineages (15) and exhibited sequence length / BLASTn scores

237 above lineage-specific thresholds. For several taxonomic groups, we constructed phylogenetic

238 trees to assess the performance of clustering, sequence quality of singletons, accuracy of OTU

239 separation, and taxonomic assignments (Fig. S1). In the course of this project, we provided 
24010,232 third-party taxonomic re-annotations to INSDC sequences to improve subsequent

241 identification of fungal sequences and made these available through the UNITE database.

243 Statistical analyses

244 Estimates of the mean annual temperature (MAT), mean annual precipitation (MAP), soil

245 moisture, and soil carbon at 30 arc second resolution were obtained from the WorldClim

246 database (www.worldclim.org). Estimates of potential evapotranspiration (PET) and net

247 primary productivity (NPP) at 30 arc minute resolution were obtained from the Atlas of the

248 Biosphere (www.sage.wisc.edu/atlas/maps.php). Variation coefficients for MAT and MAP

249 were computed based on the average monthly values to represent seasonality of temperature

250 and precipitation. We also calculated the difference of MAP to PET to evaluate the effect of

251 rainfall surplus or deficit. Based on vegetation type and geographical distribution, sites were

252 categorized into biogeographic regions and biomes following the classification of the World

253 Wildlife Foundation (http://worldwildlife.org) with a few exceptions: i) temperate deciduous

254 forests in the Northern and Southern hemispheres were treated separately; ii) tropical montane

255 forests ( $>1500 \mathrm{~m}$ elevation) were separated from the tropical lowland moist forests; and, iii)

256 grasslands and shrublands of all geographic origins were pooled. At each site, we also

257 determined the age of vegetation, time since the last fire, and EcM plant species along with

258 their relative contribution to stand basal area. EcM plants are usually conspicuous trees or

259 prominent shrubs that are relatively easy to identify and their mycorrhizal status is verifiable in

260 the field using root excavation and microscopy. Complete lists of tree species were available

261 for $<10 \%$ of the sites, so we did not directly include plant community composition parameters

262 in our analyses (but see below). 
264 using GC-combustion coupled to isotope-ratio mass spectrometry (16). Concentrations of soil

265 calcium, potassium, magnesium, and phosphorus were determined as in Tedersoo et al. (16).

266 Soil $\mathrm{pH}$ was measured in $1 \mathrm{~N} \mathrm{KCl}$ solution.

267 For analyses of fungal richness, we calculated residuals of OUT richness in relation to

268 the square root of the number of obtained sequences to account for differences in sequencing 269 depth. This method outperformed the commonly used rarefaction to the lowest number of 270 sequences method, which removes most of the data (17). We also calculated the richness of 271 major class-level taxonomic and functional groups (comprising $>100$ OTUs). We excluded

272 outlying samples dominated by a few OTUs of molds, which are indicative of poor sample

273 preservation (relative abundance of sequences belonging to Trichocomaceae $>5 \%$,

274 Mortierellaceae $>20 \%$, or Mucoraceae $>20 \%$, that exceeded three times the mean + standard

275 deviation). Although these samples were fairly homogeneously distributed across the world, 276 they had conspicuously lower fungal richness. We also excluded samples that yielded less than 2771200 sequences per sample.

278 To determine the relationship between plant and fungal richness, we relied on co279 kriging values from the global vascular plant species richness dataset (18), which covered $28096.7 \%$ of our sites. These scale-free values of plant richness were then regressed with residuals 281 from the best fit models for fungal richness and fungal functional groups. We further calculated 282 the ratio of relative plant richness to fungal richness and fitted this ratio with latitude using 283 polynomial functions to test the assumed uniformity of plant-to-fungal richness ratios at the 284 global scale $(1,19,20)$. To account for potential latitudinal biases in plant-to-fungal diversity 285 estimates, we took into account the non-uniform distribution of land surfaces by calculating an 
286 Inverse Distance Weighting (IDW) spatial interpolation of standardized ratios of plant-to-

287 residual fungal diversity using the gstat package in R (21). We then used IDW to interpolate

288 total fungal diversity beyond sampling sites, by accounting for MAP as based on the best-

289 fitting multiple regression model.

290 Distance from the equator, altitude, age of vegetation, time since last fire, climatic

291 variables, and concentrations of nutrients were log-transformed prior to analyses to improve

292 the distribution of residuals and reduce non-linearity. To account for potential autocorrelation

293 effects, we calculated spatial eigenvectors using SAM ver. 4 (22). To determine the best

294 predictors of global fungal diversity, we included edaphic, climatic, floristic, and spatial

295 variables in multiple regression models. Due to the large number of predictors, we pre-selected

29616 candidate predictors that were revealed by exploratory multiple linear and polynomial

297 regression analyses, based on coefficients of determination and forward selection criteria. The

298 most parsimonious models were determined based on the corrected Akaike information

299 criterion (AICc), which penalizes over-fitting. Finally, components of the best models were

300 forward-selected to determine their relative importance as implemented in the packfor package 301 in R.

302 To test the direct effects of climatic variables on richness of fungi and their functional

303 groups, and indirect climatic effects (via soil nutrients and vegetation), we used Structural

304 Equation Modeling (SEM) in Amos ver. 22 (SPSS Software, Chicago, IL, USA). Model fits

305 were explored based on both chi-square test and Root Mean Square Error of Approximation

306 (RMSEA). First, we included all potentially important variables (inferred from both the

307 multiple regression models and correlations for individual response variables to construct

308 separate SEM models. We tested all direct and indirect relations between exogenous and 
endogenous variables including their error terms. Then, we used backward elimination to

310 remove non-significant links to maximise whole model fit. Finally, we combined the obtained

311 SEM models in a unified path model, following the same elimination procedure.

312 In addition to full models, we specifically tested the relationships between OTU

313 richness and distance from the equator and soil $\mathrm{pH}$, because these or closely related variables

314 were usually among the most important predictors. For these analyses, we calculated residuals

315 of richness that accounted for other significant variables of the best models. To address non-

316 linear relationships, we fitted up to fifth order polynomial functions and selected best fit

317 models based on AICc values.

318 The relative effects of climatic, edaphic, spatial, and floristic variables on the total

319 fungal community composition and on particular functional groups were determined using

320 Hellinger dissimilarity (calculated if $>90 \%$ sites were represented by $>1$ shared OTUs),

321 exclusion of all OTUs that occurred once, and a multi-stage model selection procedure as

322 implemented in the DISTLM function of Permanova+ (www.primer-e.com/permanova.htm).

323 Considering computational requirements, 15 candidate variables were pre-selected based on

324 unifactorial (marginal test based on largest $F_{\text {pseudo }}$ values) and multifactorial (forward selection)

325 models. Spatial eigenvectors were not included in these analyses, because they were typically

326 of minor importance in variation partitioning analyses (see below), and to avoid making the

327 models computationally prohibitive. Optimal models were selected based on the AICc. To

328 obtain coefficients of determination (cumulative $\mathrm{R}_{\text {adjusted }}^{2}$ and statistics ( $\mathrm{F}_{\text {pseudo }}$ and P-values)

329 for each variable, components of the best models were forward selected. In parallel, we

330 prepared Global Nonmetric Multidimensional Scaling (GNMDS) graphs using the same

331 options. Significant variables were fitted into the GNMDS ordination space using the envfit 
332 function in the vegan package of R. We also grouped all climatic, edaphic, spatial, and floristic

333 variables into a variation partitioning analysis by integrating procedures in the vegan and

334 packfor packages of $\mathrm{R}$. Besides group effects, variation partitioning estimates the proportion of

335 shared variation among these groups of predictors.

336 For global biogeographic analyses, we excluded OTUs from the order Hypocreales and

337 family Trichocomaceae (both Ascomycota), because the ITS region provides insufficient

338 taxonomic resolution and known biological species are grouped together within the same OTU

339 (23). We tested the differences among fungal taxonomic and functional groups for the

340 occurrence frequency (number of sites detected) and latitudinal range of OTUs using a non-

341 parametric Kruskal-Wallis test and Bonferroni-adjusted multiple comparisons among mean

342 ranks. To test the validity of Rapoport's rule in soil fungi, we calculated the average latitudinal

343 range of OTUs for each site (24). The average latitudinal range was regressed with the latitude

344 of study sites by polynomial model selection based on the AICc criterion. This analysis was

345 run with and without OTUs only detected at a single site (range $=0$ ). Because the results were

346 qualitatively similar, we report results including all OTUs. To construct biogeographic

347 relationships among major regions and biomes, we generated cross-region and cross-biome

348 networks based on the number of shared OTUs. We excluded occurrences represented by a

349 single sequence per site. Ward clustering of biogeographic regions and biomes were

350 constructed using the Morisita-Horn index of similarity, which is insensitive to differences in

351 samples size, by use of the pvclust package of R. In this procedure, P-values are inferred for

352 non-terminal branches based on multiscale bootstrap resampling with 1,000 replicates. 


\section{Results and Discussion}

Taxonomic and functional diversity

357 Pyrosequencing analysis of global soil samples revealed 1,019,514 quality-filtered sequences 358 that were separated into 94,255 species-level OTUs (see supplementary information).

359 Altogether 963,458 (94.5\%) sequences and 80,486 (85.4\%) OTUs were classified as Fungi.

360 Most other taxa belonged to animals (Metazoa, 3.3\%), plants (Viridiplantae, 3.1\%), alveolates

361 (Alveolata, 2.8\%), and amoebae (mostly Rhizaria, 1.3\%). Kingdom-level assignment of 3.8\%

362 OTUs remained elusive. The fungal subset included 35,923 (44.6\%) OTUs that were

363 represented by a single sequence; these were removed from further analyses to avoid

364 overestimating richness based on these potentially erroneous sequences (25). The remaining

36544,563 non-singleton fungal OTUs in our data set numerically correspond to approximately

366 half of the described fungal species on Earth (1). For comparison, there are currently 52,481

367 OTUs based on $98.0 \%$ similarity clustering of all fungal ITS sequences in publicly available

368 databases (12). Global soil sampling revealed representatives of all major phyla and classes of

369 Fungi. Of fungal taxa, Basidiomycota (55.7\%), Ascomycota (31.3\%), Mortierellomycotina

370 (6.3\%) and Mucoromycotina (4.4\%) encompassed the largest proportion of sequences (Fig. 2),

371 whereas the most OTU-rich phyla were the Ascomycota (48.7\%), Basidiomycota (41.8\%),

372 Chytridiomycota (2.3\%), and Cryptomycota (syn. Rozellida; 2.1\%) (Fig. S2; Data S1). Except

373 for the recently described phylum Cryptomycota (26), the relative proportions of major phyla

374 correspond to the proportional distribution of taxa described and sequenced to date (12,

375 www.indexfungorum.org). Below the phylum level, approximately $6 \%$ of all fungal OTUs

376 could not be assigned to any known class of fungi. Further clustering of unidentified fungal 
377 sequences at $70 \%$ sequence similarity revealed 14 distinct taxonomic groups comprising $>7$

378 OTUs, suggesting that there are several deeply divergent class-level fungal lineages that have 379 not yet been described or previously sequenced.

380 Our classification revealed that $10,801(24.2 \%)$ fungal OTUs exhibited $>98 \%$ sequence 381 similarity, and $33.8 \%$ exhibited $>97 \%$ similarity, to pre-existing ITS sequences in public 382 databases. This is consistent with Taylor et al. (19), reporting 48\% of OTUs amplified from 383 Alaskan soils with $>97 \%$ similarity to any database sequences. In our study, only 4353 fungal 384 OTUs (9.8\%) were matched to sequences from herbarium specimens or fully described culture 385 collections at $>98.0 \%$ sequence similarity. Although many type collections are yet to be 386 sequenced, the paucity of matches to database entries indicates that a majority of soil387 inhabiting fungal taxa remain undescribed (19-20). These results highlight the current lack of 388 data from understudied tropical and subtropical ecosystems. The phenomenon of high cryptic 389 diversity and low success in naming OTUs at the genus or species level have been found in 390 other groups of soil microbes and invertebrates, emphasizing our poor overall knowledge of 391 global soil biodiversity (27-28).

392 The main fungal phylogenetic and functional groups were present in all ecosystems, but 393 their relative proportions varied several-fold across biomes (Figs. 2, S2-S4). The ratio of 394 Ascomycota to Basidiomycota OTUs was highest in grasslands and shrublands (1.86) and 395 tropical dry forests (1.64) but lowest in the temperate deciduous forests $(0.88)$.

396 Chytridiomycota, Cryptomycota, and Glomeromycota were relatively more diverse in the 397 grasslands and shrublands, accounting for $4.6 \%, 3.6 \%$, and $1.4 \%$ of OTU richness, 398 respectively. The relative OTU richness of Mortierellomycotina and Mucoromycotina 399 (including most fast-growing molds but also some plant symbionts) peaked in the tundra biome 
$400 \quad(4.8 \%$ and $2.7 \%$, respectively), but their abundance was lowest in tropical dry forests $(1.0 \%$

401 and $0.6 \%$, respectively). Archaeorhizomycetes, a recently described class of Ascomycetes from

402 a boreal forest (29), was most diverse in tropical moist and montane forests, particularly in

403 northern South America and New Guinea.

$404 \quad$ Among all fungal taxa, OTUs assigned to saprotrophs, EcM mutualists, and plant

405 pathogens comprised 19,540 (43.8\%), 10,334 (23.2\%), and 1770 (4.0\%), respectively (Fig.

406 S4). Other trophic categories were contained $<1 \%$ of remaining OTUs. EcM fungi contributed

$40734.1 \%$ of all taxa in the northern temperate deciduous forests, but accounted for a relatively

408 low proportion (11.9\%) in grasslands and shrublands, reflecting the paucity of host plants in

409 these ecosystems. Similarly, the proportion of EcM fungal taxa was lowest in northern South

410 America (8.0\%), where AM trees often dominate. Plant pathogens were relatively more

411 abundant and diverse in lowland tropical moist (6.2\%) and dry (6.3\%) forests.

412

413 Predictors of global richness

414 Structural equation models revealed that climate has both a strong direct effect on plant and

415 fungal richness and functional groups, but it also indirectly affects these metrics by altering

416 edaphic conditions (Main text; Fig. S5). Both SEM and regression models suggest that the best

417 predictors of diversity differed among phylogenetic and functional groups of fungi. Positive

418 effects of mean annual precipitation (MAP) and soil Ca concentration were the strongest

419 predictors of total fungal diversity, explaining $7.2 \%$ and $8.9 \%$ of residual richness, respectively

420 (Table S2). Richness of EcM fungi responded positively to the relative proportion and species

421 richness of EcM plants (explaining 18.3\% and 8.5\% of variance, respectively), as well as soil

$422 \mathrm{pH}(13.0 \%)$. EcM host species richness (5.9\%) and soil $\mathrm{pH}(20.4 \%)$ remained the strongest 
423 predictors in the best model for sites with EcM vegetation accounting for $>60 \%$ of basal area, a

424 critical point above which the proportion of EcM plants had no further effect on EcM fungal

425 richness. MAP had a strong positive effect (14.8\%) on richness of saprotrophs. Diversity of

426 plant pathogens declined with increasing distance from the equator (17.8\%) and soil $\mathrm{C} / \mathrm{N}$ ratio

427 (11.6\%). Animal parasites responded positively to MAP (20.3\%), whereas monthly variation of

428 precipitation (MAP CV) had a negative impact on richness of mycoparasites (fungus-parasitic

429 fungi; 8.2\%). Richness of the AM Glomeromycota was negatively related to the age of

430 vegetation (7.3\%) but positively related to potential evapotranspiration (PET, 3.5\%) and soil

$431 \mathrm{pH}(4.3 \%)$. Of the major taxonomic groups, the richness of Ascomycota in general (18.5\%)

432 and that of Archaeorhizomycetes (21.7\%) were negatively related to distance from the equator

433 in best-fit models. Climatic variables were the best predictors for richness of

434 Mortierellomycotina (MAT: negative effect, 26.1\%) and the ascomycete classes

435 Dothideomycetes (MAT: positive effect, 20.9\%), Lecanoromycetes (MAT: negative effect,

436 26.7\%), Leotiomycetes (MAT: negative effect, 30.1\%), Orbiliomycetes (MAT: positive effect,

437 12.8\%), and Sordariomycetes (MAP: positive effect, 33.4\%). The richness of Chytridiomycota

438 and the ascomycete class Pezizomycetes was best explained by a positive response to soil $\mathrm{pH}$

439 (8.6\% and $40.5 \%$, respectively). Concentration of soil nutrients or their ratio to other nutrients

440 were the strongest predictors for OTU richness of Cryptomycota ( $\mathrm{N}$ concentration: positive

441 effect, 10.1\%), Geoglossomycetes (N/P ratio: positive effect, 3.7\%), Mucoromycotina (C/N

442 ratio: positive effect, 19.0\%), and Wallemiomycetes (P concentration: negative effect, 14.9\%).

443 The richness of Basidiomycota and its class Agaricomycetes were best explained by a positive

444 response to soil Ca concentration (13.5\% and $12.8 \%$, respectively). 
Although geographical distance per se had negligible effects on richness (Moran's

$446 \mathrm{I}=0.267)$, spatial predictors were included in the best richness models of nearly all functional

447 and phylogenetic groups (except Glomeromycota), indicating regional- or continental-scale

448 differences in OTU richness (Fig. 1B). Compared to other tropical regions, richness of fungi

449 was conspicuously lower in Africa, independent of biome type. These results might reflect the

450 relatively lower MAP in much of Africa compared with other tropical continents.

451 Alternatively, lower fungal richness could be related to the disproportionately strong shifts in

452 biomes during the Pleistocene, which impoverished the African flora (18).

453 Among edaphic variables, soil $\mathrm{pH}$ and Ca concentration were typically the most

454 important predictors of fungal OTU richness. These variables positively correlated with fungal

455 richness at the global scale $\left(\mathrm{F}_{1,335}=290.7 ; \mathrm{R}_{\text {Pearson }}=0.682 ; \mathrm{P}<0.001\right)$. The strong positive

456 influence of soil Ca concentration on richness of fungi, in particular Basidiomycota, is

457 congruent with a similar positive relationship found for $\mathrm{Ca}$ and EcM fungal richness associated

458 with Northern Hemisphere Alnus spp. (30). Exchangeable Ca is important for many

459 physiological processes in plants and microorganisms and it influences the turnover rate of soil

460 organic matter (31). In soil geochemical processes, $\mathrm{pH}$ and Ca concentration affect each other

461 and thus may have both direct and indirect effects on soil biota. Fungal functional groups were

462 differentially affected by $\mathrm{pH}$. Richness of EcM fungi was greatest in slightly acidic to neutral

463 soils (Fig. S6), whereas saprotrophs, especially white rot decomposers, were more diverse in

464 moderately to strongly acidic soils. Richness of Pezizomycetes peaked distinctly in neutral

465 soils.

466

467 Macroecological patterns 
468 In general agreement with biogeographic patterns of plants, animals, and foliar endophytic

469 fungi $(5,32)$, the overall richness of soil fungi increased towards the equator (Fig. 3A).

470 However, major functional and taxonomic groups showed dramatic departures from the

471 general latitudinal richness patterns (Figs. 3, S7). Namely, diversity of saprotrophic fungi,

472 parasites, and pathogens increased at low latitudes, whereas richness of EcM fungi peaked at

473 mid-latitudes, especially in temperate forests and Mediterranean biomes of the Northern

474 Hemisphere $\left(40-60^{\circ} \mathrm{N}\right.$; Fig. S8). By contrast, saprotrophic fungi had a broad richness peak

475 spanning from ca. $45^{\circ} \mathrm{S}$ to $25^{\circ} \mathrm{N}$. Richness of Ascomycota, in particular that of

476 Archaeorhizomycetes, Dothideomycetes, Eurotiomycetes, Orbiliomycetes, and

477 Sordariomycetes, peaked in tropical ecosystems (Fig. S7). Conversely, the ascomycete classes

478 Lecanoromycetes and Leotiomycetes as well as Microbotryomycetes (basidiomycete yeasts),

479 Mortierellomycotina, and Mucoromycotina increased in diversity towards the poles, with no

480 noticeable decline in boreal forests and tundra biomes. Agaricomycetes, Pezizomycetes, and

481 Tremellomycetes exhibited distinct richness peaks at mid-latitudes. Richness of

482 Agaricomycetes was greater in the Northern Hemisphere, whereas that of Microbotryomycetes,

483 Tremellomycetes, and Wallemiomycetes peaked in the Southern Hemisphere temperate

484 ecosystems (Fig. S8).

485 All of these phylogenetic groups originated $>150$ million years ago on the

486 supercontinent Pangaea (33) and have had sufficient time for long-distance dispersal. However,

487 our data suggest that particular regional biotic or abiotic conditions (e.g., soil $\mathrm{pH}$ and favorable

488 climatic conditions) have likely stimulated evolutionary radiations in certain geographic areas

489 and not in others. Adaptation to cold climate in younger fungal phyla has been suggested to

490 explain differential latitudinal preferences among fungal groups (34). However, our global 
491 analysis provided no support for this hypothesis (Fig. S9). Instead, it revealed that ancient

492 lineages are relatively more common in non-wooded ecosystems.

494 Relation of plant and fungal richness

495 Plant and fungal richness were positively correlated (Fig. S10), but plant richness explained no

496 residual richness of fungi based on the best regression model $\left(\mathrm{R}^{2}{ }_{\mathrm{adj}}<0.01 ; \mathrm{P}>0.05\right)$. These

497 results and SEM path diagrams suggest that correlations between plant and fungal richness are

498 best explained by their similar response to climatic and edaphic variables (i.e., covariance)

499 rather than by direct effects of plants on fungi. However, when separating functional

500 categories, trophic groups of fungi exhibited differential response to plant diversity and relative 501 proportion of potential hosts.

502 Plant pathogens usually attack a phylogenetically limited set of host plants (35),

503 suggesting that that plant pathogens have at least partly co-evolved with their hosts and may

504 have radiated more intensively in the tropics where high plant diversification and richness

505 permit greater diversification. Strong phylogenetic signals in soil feedbacks, adaptive radiation,

506 and negative density dependence (the Janzen-Connell hypothesis) have probably contributed to

507 the pronounced richness of both plants and their pathogens at low latitudes $(36,37)$. However,

508 our analyses revealed no significant effects of plant richness per se on residual richness of

509 pathogens in soil. Similarly to pathogens, richness of AM fungi was unrelated to the proportion

510 of AM host trees or interpolated host richness, which may result from non-specific associations

511 with tree and understory species. Hence both AM and soil pathogen richness were unaffected

512 by plant richness. By contrast, host richness explained 6\% of variation in EcM fungal richness,

513 indicating either niche differentiation of fungi in forests of mixed hosts or sampling effects 
514 (i.e., forests with higher host diversity are more likely to include plant species that harbor high

515 fungal diversity). With a few notable exceptions, most studies have found low levels of host

516 preference or host specificity among EcM fungi (38). We found that relative EcM host density

517 had a strong influence on EcM fungal richness, suggesting that greater availability of

518 colonizable roots in soil provides more carbon for EcM fungi and thereby yields greater

519 species density and local-scale richness regardless of latitude. The peak of EcM fungal

520 taxonomic and phylogenetic richness in northern temperate biomes coincides with the

521 geographical distribution and dominance of Pinaceae, which is the oldest extant EcM plant

522 family $(15,39)$.

523 The ratio of plant-to-fungal richness decreased exponentially with increasing latitude,

524 because plant diversity dropped precipitously toward the poles relative to fungal diversity (Fig.

525 4). This finding calls into question present global fungal richness estimates. These estimates

526 assume similar spatial turnover of plant and fungal species and a constant plant-to-fungus ratio,

527 and have been formulated based mostly on data from temperate and boreal ecosystems $(1,19$,

528 20). Yet local-scale beta diversity of both plants and fungi differ among temperate and tropical

529 sites $(40,41)$ and there are profound differences in plant species turnover depending on

530 propagule size (42). Natural distribution of very few vascular plant species encompass several

531 continents, but there are multiple fungal species with circumpolar or cosmopolitan distribution

532 (43, 44; see Biogeography section below). While we cannot directly compare plant and fungal

533 beta diversity, spatial turnover of plant species is inarguably greater (42). Based on the

534 function of fungi-to-plant richness ratio to latitude and latitudinal distribution of land, we

535 calculated that fungal richness is overestimated by 1.5 - and 2.5 -fold based on constant

536 temperate $\left(45^{\circ}\right.$ latitude) and boreal ( $65^{\circ}$ latitude) richness ratios, respectively. 
Since richness estimates are calculated based on the frequency of the rarest species, the

538 reliability of singleton data call into question biologically meaningful extrapolations (11). In

539 metabarcoding studies such as ours, sequencing errors tend to give rise to singleton sequences,

540 and the number of rare artificial taxa grows rapidly with increasing sequencing depth (25).

541 Therefore, despite the size of our dataset, it cannot readily be used to produce reliable

542 taxonomic richness extrapolations.

543

544 Community ecology

545 Variation partitioning analysis revealed that climatic, edaphic, and floristic variables (and their

546 shared effects) are the strongest predictors for community composition of all fungi and most of

547 their functional groups (Fig. S11). However, the saprotroph community composition was most

548 strongly explained by purely spatial variables. More specifically, PET and soil pH explained

$5492.4 \%$ and $1.5 \%$, respectively, of the variation in total fungal community composition (Table

550 S3; Fig. S12). PET contributed 3.8\%, 2.8\%, and 11.7\% to community structure of saprotrophs,

551 plant pathogens, and yeasts, respectively. Distance from the equator $(1.3 \%)$ and soil $\mathrm{pH}(0.7 \%)$

552 were the strongest predictors of EcM fungal community composition, whereas mean annual

553 temperature (4.0\%) was the strongest predictor for animal parasites, and distance from the

554 equator (3.5\%) was the best predictor for mycoparasites (Table S3; Fig. S12).

555

These results indicate that both environmental and spatial predictors generally have a

556 minor influence on species-level composition of fungi at the global scale. Nonetheless, the

557 significant global-scale $\mathrm{pH}$ effect in several groups of fungi is consistent with the substantial

558 influence of $\mathrm{pH}$ on the phylogenetic structure of soil fungal and bacterial communities in both

559 local and continental scales $(27,45)$. The relatively stronger climatic and edaphic drivers of 
560 richness at the class and phylum level suggest that phylogenetic niche conservatism in fungal

561 lineages is similar to cross-biome distribution patterns in vascular plants (46) and protists (47).

563 Global biogeography

564 Consistent with Rapoport's rule formulated for macro-organisms (24) and later applied to 565 marine bacteria (48), the mean latitudinal range of fungi strongly increased towards the poles

566 (Fig. S13). These results also suggest that a greater proportion of fungi are endemic within

567 tropical rather than extra-tropical ecosystems.

$568 \quad$ Major taxonomic and functional groups of fungi differed markedly in their distribution 569 range (Figs. S14, S15). Animal parasites were more widely distributed compared with all other 570 groups, suggesting that there are many generalist OTUs with global distribution. Saprotrophs 571 and plant pathogens had broader distribution ranges than EcM and AM root symbionts. Taxa

572 belonging to Mortierellomycotina, Mucoromycotina, Tremellomycetes, and Wallemiomycetes 573 - groups that include a large proportion of saprotrophs and parasites that produce exceptionally

574 large quantities of aerially dispersed mitospores - were generally most widely distributed.

575 Besides the AM Glomeromycota, OTUs belonging to the ascomycete classes

576 Archaeorhizomycetes, Geoglossomycetes, and Orbiliomycetes were detected from the fewest

577 sites.

578 The northernmost biogeographic regions (Europe, West Asia, East Asia, and North

579 America) had the most similar fungal communities as revealed by shared fungal OTUs (Fig. 5).

580 Based on the Morisita-Horn similarity index, the northern and southern temperate regions

581 clustered together with marginally non-significant support ( $\mathrm{P}=0.064$; Fig. 6A). In spite of the

582 large geographical distance separating them, paleo- and neotropical biogeographic regions 
583 clustered together $(\mathrm{P}=0.059)$. However, biogeographic clustering of regions deviated markedly

584 in certain functional groups of fungi (Fig. 6). For instance, EcM fungi in the southern

585 temperate and tropical regions had greater similarity compared with northern temperate

586 ecosystems $(\mathrm{P}=0.001)$. Among biomes, boreal forests, temperate coniferous forests, and

587 temperate deciduous forests shared the largest numbers of fungal OTUs (Fig. S16). Fungal

588 OTUs in temperate deciduous forests were highly similar to Mediterranean and tropical

589 montane forests, whereas fungal OTUs in tropical montane forests were linked to tropical

590 moist forests, which in turn exhibited substantial connections with tropical dry forests and

591 savannas. As a result, cluster analysis supported separation of tropical and non-tropical biomes

592 (Fig. 6B). Consistent with biogeographic region-level analysis, lowland tropical biomes, arctic

593 tundra and boreal forests biomes, and temperate biomes formed three well-supported clusters.

594 Tropical montane forests and grasslands and shrublands were clustered with temperate biomes

595 based on distribution of all fungi and most functional groups. However in EcM fungi, taxa

596 from southern temperate forests, tropical montane forests, and grass/shrublands clustered with

597 tropical lowland and Mediterranean biomes. A relatively large proportion of EcM fungal taxa

598 were shared across various biomes in Australia and New Guinea, which explains these

599 deviating patterns. In contrast, plant pathogens from tropical montane forests clustered with

600 tropical lowland biomes rather than with temperate biomes.

601 Our biogeographic analyses complement the community-level results suggesting that

602 both climate and biogeographic history shape macro-ecological patterns of fungi. Co-migration

603 with hosts over Pleistocene land bridges (e.g., Beringia, Wallacea, Panamanian) and long-

604 distance dispersal by spores appear to have played important roles in shaping current fungal

605 distribution patterns $(30,43)$. The relative influence of climate and biotrophic associations with 
606 host plants of varying extant distributions probably contribute to differences in the range and

607 biogeographic relationships among fungal functional groups (49). In addition, taxon-specific

608 constraints for dispersal, such as shape and size of propagules and sensitivity to UV light, may

609 differentially affect long-distance dispersal among taxa (7). For instance, Glomeromycota

610 OTUs, which form relatively large non-wind dispersed asexual spores, had the lowest average

611 geographical range. In general, region-based distribution patterns of fungi are somewhat

612 conflicting with clustering of plants and animals, where Holarctic lineages are deeply nested

613 within larger tropical groups (50). Consistent with macro-organisms, fungi from the Southern

614 Hemisphere temperate landmasses cluster together. Differences observed in macro-ecological

615 patterns among fungi, plants, and animals may originate from the relative strength of dispersal

616 limitation and phylogeographic history, but exaggeration by methodological differences among

617 studies cannot be discounted. The use of homogenous sampling and analytical methods, as

618 done in this study, are necessary to confidently compare macro-ecological patterns amongst

619 distinct life forms and to reliably test degrees of consistency among all kingdoms of life.

620

621 Conclusions and perspectives

622 Climatic variables explained the greatest proportion of richness and community composition in

623 fungal groups by exhibiting both direct and indirect effects through altered soil and floristic

624 variables. The strong driving climatic forces identified here open up concerns regarding the

625 impact of climate change on the spread of disease (51) and the functional consequences of

626 altered soil microorganism communities (52). The observed abrupt functional differences

627 between fungal communities in forested and treeless ecosystems, despite spatial juxtaposition,

628 suggests that plant life form and mycorrhizal associations determine soil biochemical processes 
629 more than plant species per se. Loss of tree cover and shrub encroachment resulting from

630 drying and warming may thus have a marked impact on ecosystem functioning both above-

631 and belowground.

632 In addition to natural mechanisms, such as long-distance dispersal and migration over

633 past land bridges, global trade has enhanced the spread of some non-native soil organisms into

634 other ecosystems, where they sometimes become hazardous to native biota, economy, and

635 human health (53). Our results highlight how little insight we still have into natural microbial

636 distribution patterns, and this undermines our ability to appraise the actual role of humans in

637 shaping these biogeographic processes. Even larger-scale sampling campaigns are needed to

638 provide data for establishing natural distributions and building species distribution models

639 (52), which will enable us to predict the spread and habitat suitability of non-native

640 microorganisms.

641 
644 1. M. Blackwell, Am. J. Bot. 98, 426 (2011).

645 2. N. Fierer et al., Ecol. Lett. 12, 1 (2009).

646 3. H. Serna-Chavez, N. Fierer, P. M. van Bodegom, Glob. Ecol. Biogeogr. 10, 1162 (2013).

647 4. X. Xu, P. Thornton, W. M. Post, Glob. Ecol. Biogeogr. 22, 737 (2013).

648 5. H. Hillebrand, Am. Nat. 163, 192 (2004).

649 6. G. G. Mittelbach et al., Ecol. Lett. 10, 315 (2007).

650 7. B. J. Finlay, Science 296, 1061 (2002).

651 8. D. R. Nemergut et al., Microbiol. Mol. Biol. Rev. 77, 342 (2013).

652 9. K. G. Peay, M. I. Bidartondo, A. E. Arnold, New Phytol. 185, 878 (2010).

653 10. J. M. Talbot et al., Proc. Natl. Acad. Sci. USA, 111, 6341 (2014)

654 11. B. D. Lindahl et al., New Phytol. 199, 288 (2013).

655 12. U. Kõljalg et al., Mol. Ecol. 22, 5271 (2013).

656 13. K. Abarenkov et al., Evol. Bioinform. 6, 189 (2010).

657 14. S. M. Adl et al., J. Eukaryot. Microbiol., 59, 527 (2012).

658 15. L. Tedersoo, M. E. Smith, Fung. Biol. Rev. 27, 83 (2013).

659 16. L. Tedersoo et al., New Phytol. 195, 832 (2012).

660 17. I. Hiiesalu et al., New Phytol. 203, 233 (2014).

661 18. H. Kreft, W. Jetz, Proc. Natl. Acad. Sci. USA 104, 5925 (2007).

662 19. D. L. Taylor et al., Ecol. Monogr. 84, 3 (2014).

663 20. H. E. O'Brien et al., Appl. Environ. Microbiol. 71, 5544 (2005). 
664 21. R Core Team, R: a language and environment for statistical computing. Vienna: R

665 Foundation for Statistical Computing (2014).

666 22. T. F. Rangel et al., Ecography 33, 46 (2010).

667 23. C. L. Schoch et al., Proc. Natl. Acad. Sci. USA 109, 6241 (2012).

668 24. G. C. Stevens, Am. Nat. 133, 240 (1989).

669 25. I. A. Dickie, New Phytol. 188, 916 (2010).

670 26. M. D. M. Jones et al., Nature 474, 200 (2011).

671 27. C. Lauber et al., Appl. Environ. Microbiol. 75, 5111 (2009).

672 28. M. S. Robeson et al., Proc. Natl. Acad. Sci. USA 108, 4406 (2011).

673 29. A. Rosling et al., Science 333, 876 (2011).

674 30. S. Põlme et al., New Phytol. 198, 1239 (2013).

675 31. P. B. Reich et al., Ecol. Lett. 8, 811 (2005).

676 32. A. E. Arnold, Fung. Biol. Rev. 21, 51 (2007).

677 33. M. L. Berbee, J. W. Taylor, Fung. Biol. Rev. 24, 1 (2010).

678 34. K. K. Treseder et al., Ecol. Lett. 9, 1086 (2014).

679 35. G. S. Gilbert, C. O. Webb, Proc. Natl. Acad. Sci. USA 104, 4979 (2007).

680 36. X. Liu et al., Ecol. Lett. 15, 111 (2012).

681 37. R. Bagchi et al., Nature 506, 85 (2014).

682 38. M. Bahram et al., Fung. Ecol. 7, 70 (2013).

683 39. L. Tedersoo et al., Mol. Ecol. 21, 4160 (2012).

684 40. M. Bahram et al., J. Ecol. 101, 1335 (2013).

685 41. H. Qian et al., Glob. Ecol. Biogeogr. 22, 659 (2013).

686 42. H. Qian, Glob. Ecol. Biogeogr. 18, 327 (2009). 
43. J. Geml et al., J. Biogeogr. 34, 74 (2012).

688 44. I. Timling et al., Mol. Ecol. 23, 3258 (2014).

689 45. J. Rousk et al., ISME J. 4, 1340 (2010).

690 46. M. D. Crisp et al., Nature 458, 754 (2009).

691 47. S. T. Bates et al., ISME J. 7, 652 (2013).

692 48. W. Jun Sul et al., Proc. Natl. Acad. Sci. USA 110, 2342 (2013).

693 49. H. Sato et al., Mol. Ecol. 21, 5599 (2012).

694 50. I. Sanmartin, F. Ronquist, Syst. Biol. 53, 216 (2004).

695 51. S. Altizer et al. Science 341, 514 (2013).

696 52. W. H. van der Putten et al., Phil. Trans. R. Soc. B 365, 2025 (2010).

697 53. M.-L. Desprez-Loustau et al., Trends Ecol. Evol. 22, 472 (2007).

698

\section{Acknowledgements}

700

701 The sequence data and metadata are deposited in the Short Read Archive (accession

702 SRP043706) and UNITE databases. Data used for analyses are available as supplementary

703 online material Data S1 and S2. We thank H. Mann, D. Sveshnikov, F.O.P. Stefani, A. Voitk,

704 and Y. Wu for supplying single soil samples; R. Puusepp, M. Haugas, and M. Nõukas for

705 sample preparation; H. Kreft for providing interpolated plant diversity data; S. Jüris for

706 designing the printed figure; M.I. Bidartondo, K.G. Peay and three anonymous reviewers for

707 constructive comments on the manuscript; and relevant institutions of multiple countries for

708 issuing permissions for sampling and delivery. The bulk of this project was funded from

709 Estonian Science Foundation grants 9286, 171PUT, IUT20-30; EMP265; FIBIR; ERC; and in 
710 part by numerous funding sources that facilitated co-author efforts in collecting and pre-

711 processing samples.

712 


\section{$714 \quad$ Figure legends}

716 Fig. 1. Map of A) global sampling (circles as study sites); B) Interpolated taxonomic richness

717 of all fungi using Inverse Distance Weighting (IDW) algorithm and accounting for the

718 relationship with mean annual precipitation (based on the best multiple regression model).

719 Different colors depict residual Operational Taxonomic Unit (OTU) richness of all fungi

720 accounting for sequencing depth. Warm colors indicate OTU-rich sites, whereas cold colors

721 indicate sites with fewer OTUs.
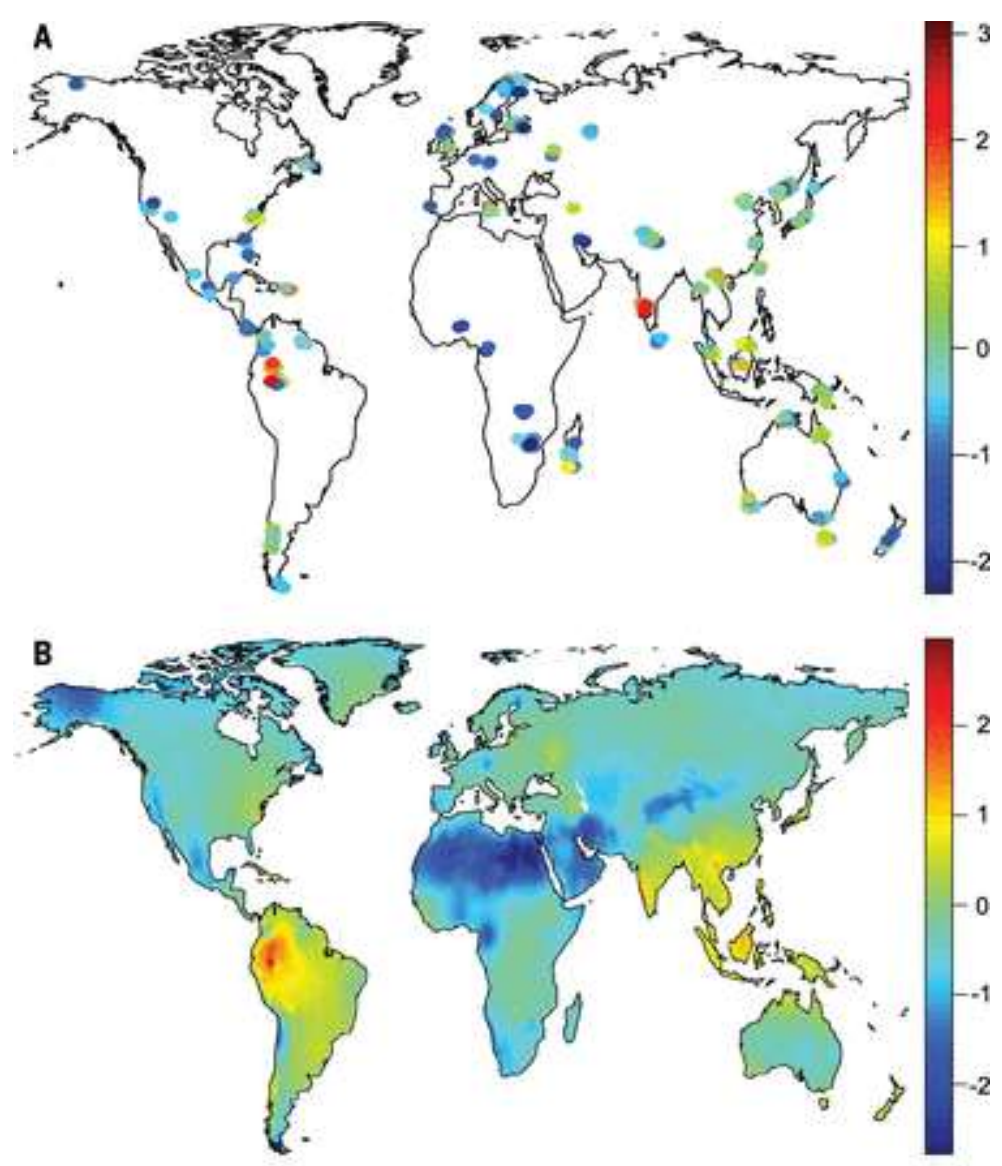

723 
724 Fig. 2. Relative proportion of fungal sequences assigned to major taxonomic groups in 725 different biomes.

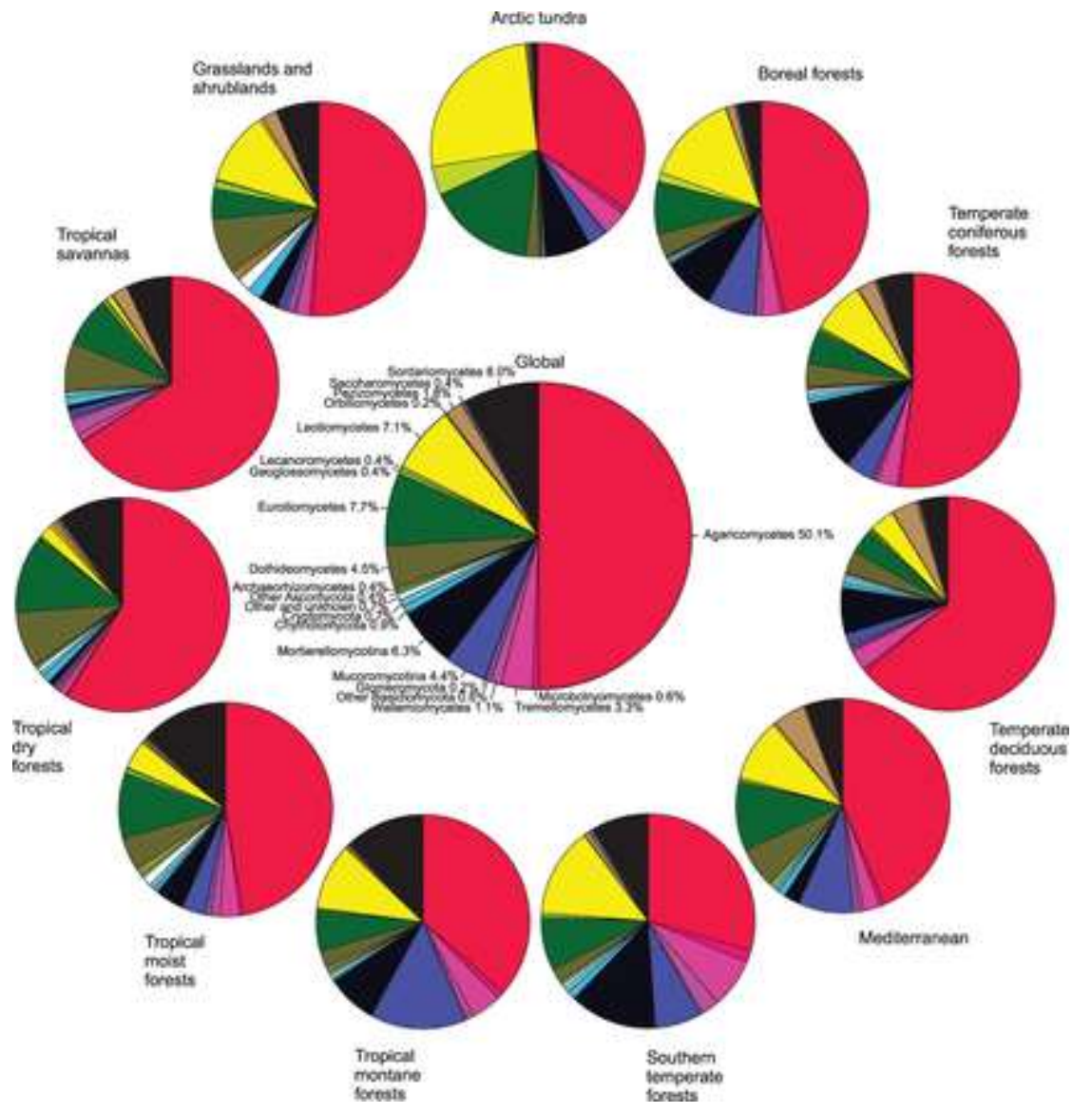


729 Fig. 3. Relationships between residual richness of fungal taxonomic or functional groups and

730 distance from the equator. A, all fungi; B, ectomycorrhizal (EcM) fungi; $\mathrm{C}$, saprotrophic fungi;

731 D, plant pathogens; E, animal parasites; F, mycoparasites; G, white rot decomposers; and H,

732 yeasts. Lines indicate best-fitting linear or polynomial functions.
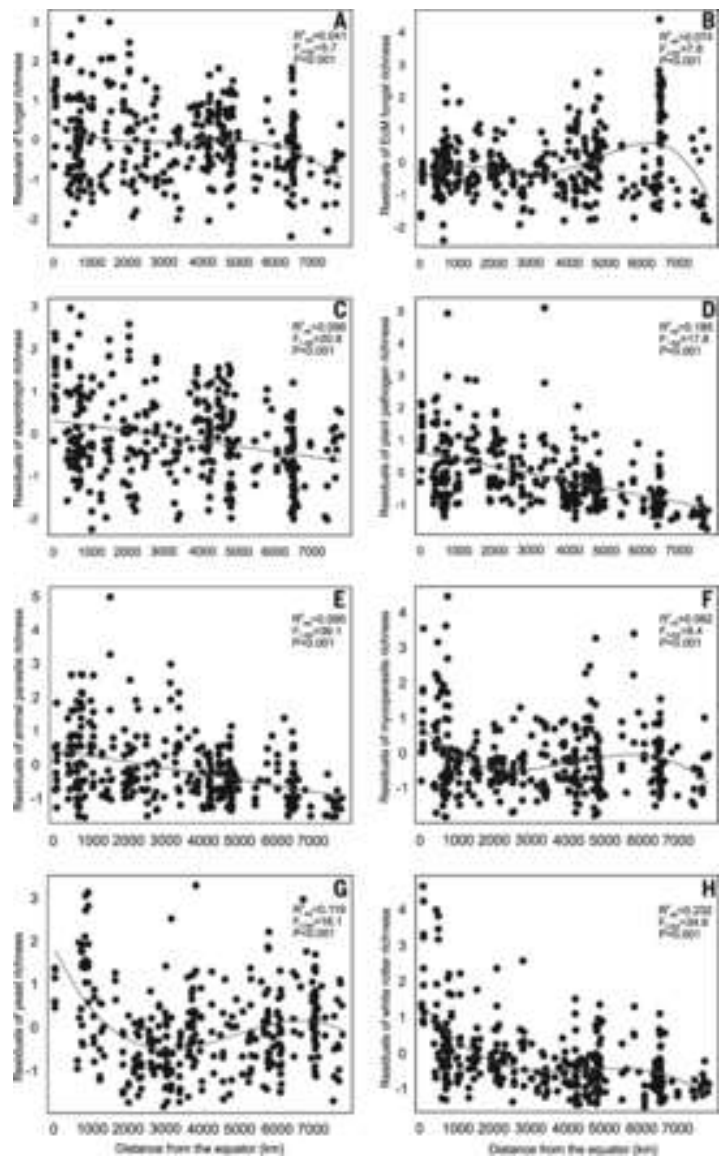

734

735 
736 Fig. 4. Relationship between standardized plant richness to fungal richness ratio and distance

737 from the equator based on (A) interpolated values and (B) polynomial regression. Residuals of

738 fungal richness are taken from the best linear regression model accounting for other significant

739 predictors. Warm colors indicate high plant-to-fungal richness ratio, whereas cold colors

740 indicate low plant-to-fungal richness.
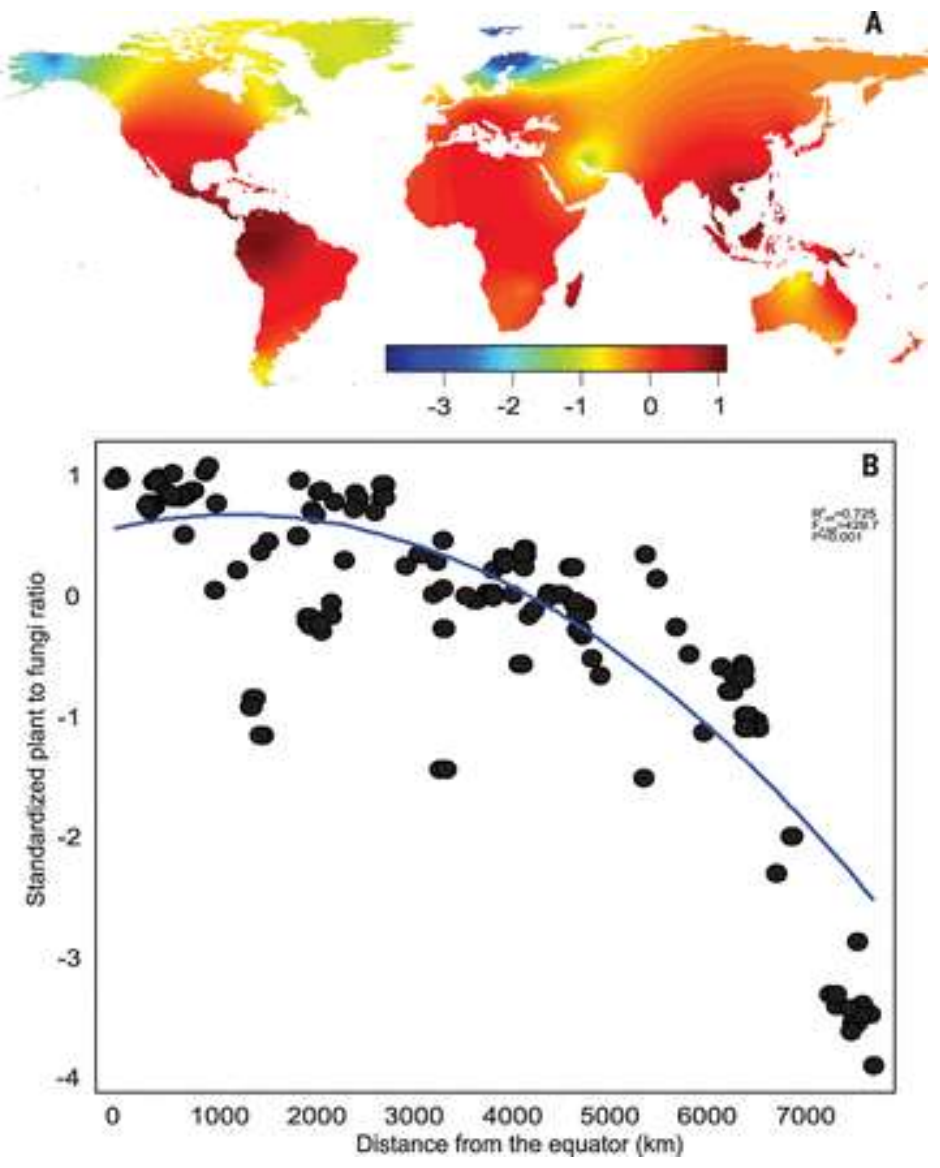
743 Fig. 5. Connectedness of biogeographic regions by shared Operational Taxonomic Units

744 (OTUs) of ectomycorrhizal fungi (blue), saprotrophs (black), and plant pathogens (red). The

745 width of lines and diameter of circles are proportional to the square root of the number of

746 connections and sample size (number of sites), respectively. Numbers in circles indicate the

747 number of OTUs found in each region. OTUs with a single sequence per site and OTUs

748 belonging to Hypocreales and Trichocomaceae (in which the ITS region is too conservative for

749 species-level discrimination) were excluded.

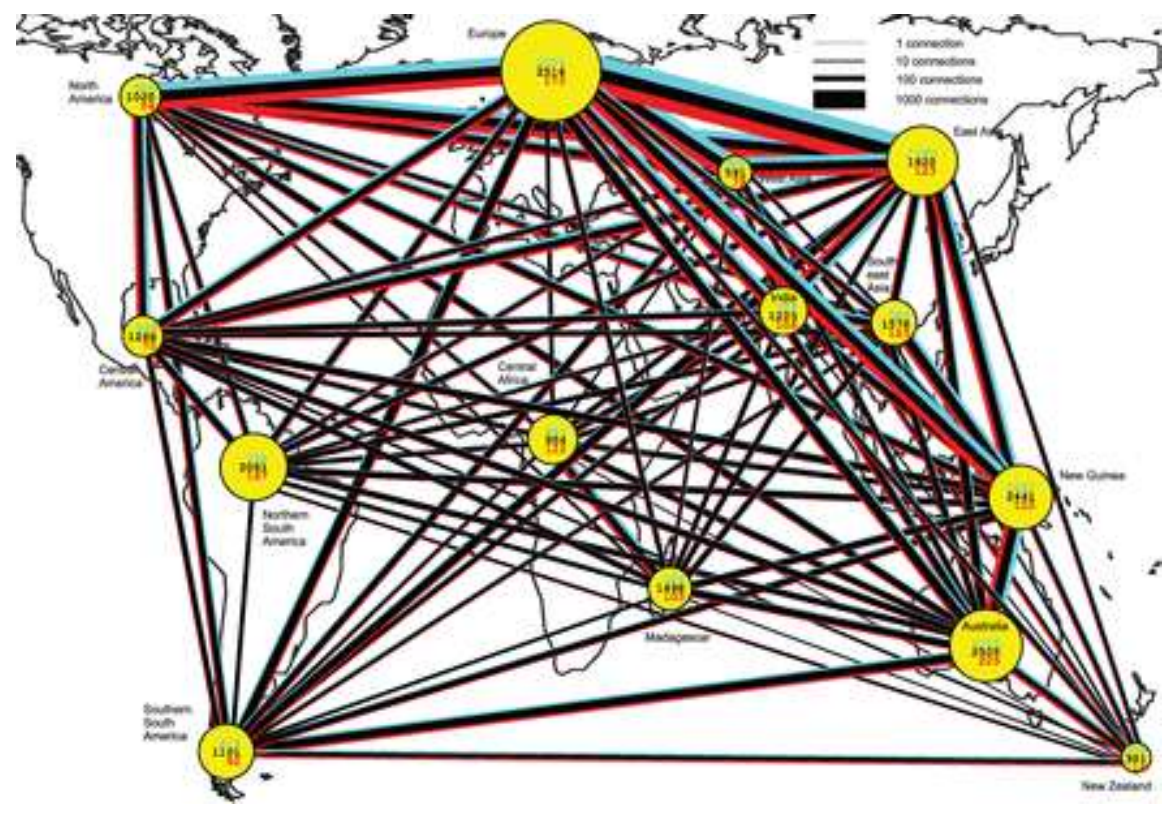


752 Fig. 6. Ward clustering of biogeographic regions (left panes) and biomes (right panes) based

753 on the Morisita-Horn pairwise similarity index in A and B, all fungi; C and D, ectomycorrhizal

754 fungi; $\mathrm{E}$ and $\mathrm{F}$, saprotrophs; $\mathrm{G}$ and $\mathrm{H}$, plant pathogens. Numbers above branches indicate $\mathrm{P}$ -

755 values.
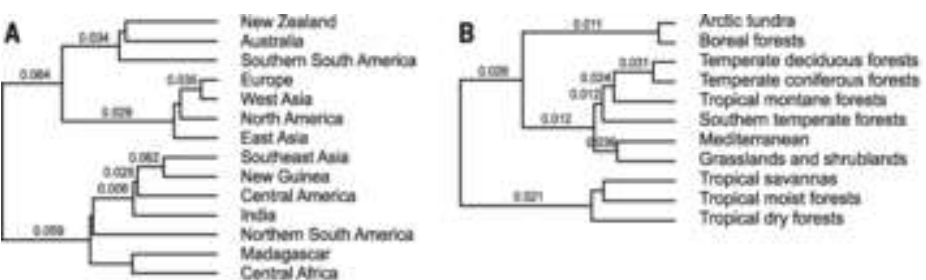

C Northern South Ameticil Nortient South America
Comral Anterica Solthem sout soultieast neen New Gunes New Zeaian Madagumcar Central Ano Weat Aatio anin $\operatorname{loms}^{2}$ Europe an

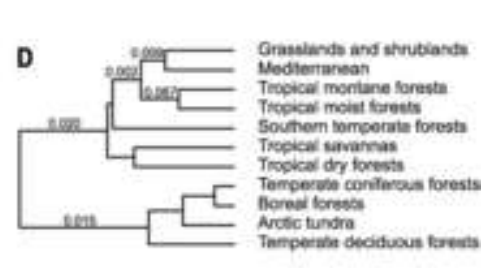
sorar $\square$ Sosthem South America F

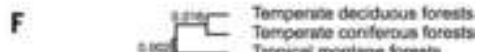

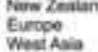
Worth Avisurica ant Aasa soltheost Asen

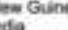
Cantral Avterica Centagancar Northers South Amenca
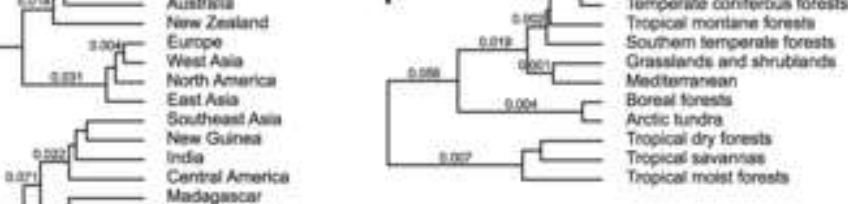

Sowthen South Anerca
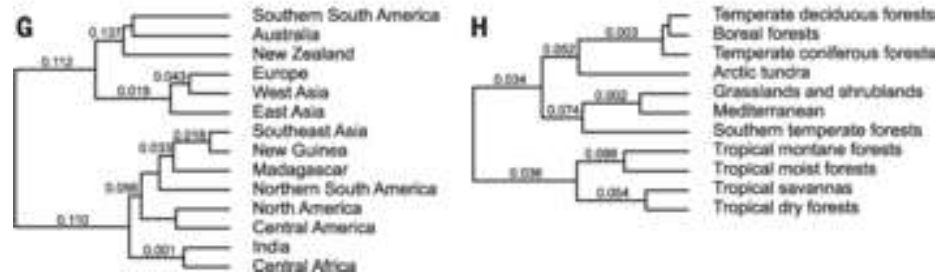
759 Supplementary Materials

760

761 Figs. S1-S16

762

763 Tables S1-S3

764

765 Data S1-S2

766 